\title{
Cloud Computing Enabled External Applications to Car Users using Nomadic Smartphones
}

\author{
L. Nkenyereye, J.W. Jang \\ Department Computer engineering \\ Dong-Eui University \\ Korea
}

\begin{abstract}
Vehicle on-boarddiagnostics and vehicle information obtained from devices mounted on roadinfrastructures, detectors located along the roadside are pivotal to Intelligent Transport Systems servicesincluding real time management of driven information. The value of data from driven information has a significant role but the way of extracting by car manufacturers, actors in automotive ecosystem, traffic and road management services differs.In fact, the car manufacturers are also prompted to deliver cloud based applications and others services to car users which should be carried out by cloud computing services layers. In this paper, we present a remote vehicle assignation services cloud computing responsible of real time analysis of vehicle information in one hand and providing internet applications to the car user in other hands by using a vehicle to applications smart phones interface approach that considers the smartphones to acts as a remote users which passes driver inputs and delivers output from external applications.
\end{abstract}

Keywords-intelligent transportation systems; cloud computing; external applications; vehicle-to-application; nomadic smartphones; vehicle on-board diagnostics

\section{INTRODUCTION}

Mobile phones, portable navigation units known as Nomadic Information and communications Technologies devices [1] are now days being used within the automotive cockpit [2] by car owners or drivers while driving their vehicles. Automotive industry has recently deployed enough resources to build efficient cars that are most comfortable, equipped by several functions which will allow car's owner dealing with all interested in automotive ecosystem such as vehicle manufacturing[3], vehicle repair, Vehicle Dealer ship management, vehicle logistics, emergency services, insurance companies, then benefit several services from them. Moreover, recently, we have witnessed the revolution observed in Information technology that implicates a huge number of standardized information and communications technology capability such as software, Information technology infrastructure afforded by different services providers that are accessible anywhere via Internet, always available to the end user when he needs them, making collection of data from several sensors units, facilitating reliable processing and easier storage of data collected. Those IT capabilities are faster and more economical. However, some car manufacturers have developed their own applications data centers to collect more data which have tasks to provide cloud based applications to car users [4]. The connection based method is essential as it consists of catching information using Bluetooth, $3 \mathrm{G}$ or $4 \mathrm{G}$
LTE technologies via smartphones. Cloud computing platform is absolutely essential for collecting vehicle information, implementing and deploying external applications to car owners. It set overs an integration of services with which to realize an efficient and accuracy information exchange between vehicles and remotely web services, and points out the potential of the connected car context. Vehicle to applications handles a multitude of applications as for instance, monitoring performance of vehicles sold in the market by leveraging reporting of Diagnostic Troubles codes (DTC), status diagnosis information, emergency services management to locate and help victims of accidents or injured person, traffic transportation authorities to increase safety, accident prevention, car repair to analyze vehicle diagnosis in real time while the vehicle breaks down.

In the context that the presence of using nomadic devices while drivers operating the car has managed to connect car to others devices within the car/vehicles, networks and services outside the car, smartphones features dashboard with a screen from which the operations of the connections should be handled by the driver. Beside of smartphonesfeatures, Vehicleto-vehicle and vehicle-to-infrastructure involved carrying out the interconnectivity and communication between ad-hoc vehicular networks (VANETs) [5] and the networks are not fully integrated into vehicles, CAN protocols diversify from one car manufacturers which points out a wide obstacle for establishment of external applications using in-vehicle gatewayplatform [6] which is not integrated in all vehicles.According to those challenges previously described, a vehicle to applications smartphonesinterface approach that considers the vehicle to acts as a remote users which passes driver inputs and delivers output from external applications is proposed in this paper to deliver external applications and other services offered by actors in automotive ecosystem to the car owners. Moreover, uncertainty, unreliable world widely identification of vehicle's owner by all interested in automotive industryconstitutes also a challenge. However, in order to deal with data from vehicle diagnostics data and offered external applications and services, a scale computing that features real time analysis, integration, monitoring and security process is proposed in this paper. Cloud computing can be featured into private and public cloud [7]. Cloud computing provides processing and management platform, allows services made available to users on demand via internet from a cloud computing provider's servers [8]. Cloud providers are designed to offer a scalable access to applications, services and resources. 


\section{EXISTING WORK}

\section{A. Cloud Computing for Vehicles}

Major Benefits of cloud computing for vehicles relies on safety, real time vehicle services with special focus on customized services like resource sharing, parking [9]. ITS value added services [10] are defined in the E-FRAME project that its primary aim is to describe the extensions to the user's car needs [11].Applications included in Value-added and others services are for instance Remote Diagnosis and Just In Time RepairNotification, Automatic Access Control/Parking Access, Map Downloading and update described respectively in Vehicle SupportManagement, Parking Management, Enhanced Route Guidance Map Downloading and updateand Navigation service/category topic[11]. External application/service refers to applications/services that were not integrated into the vehicle at the time of manufacture. Such applications/services include those that reside on a nomadic device brought into the vehicle, roadside station, or cloud-based server. [12]

\section{PROPOSED CLOUD COMPUTING ENVIRONMENT}

\section{A. Remote Vehicle Assignation Service Cloud Computing (RVASCC)}

The RVMCC reports multiple services to support ITS value add service and enable actors in automotive ecosystem provide services. First our service model suggests a group if ITS value added service. Second, the third party aims to have access to a reliable data with appropriate computing analysis. The remote service event processing will be explained in the next section. Finally, the IaaS based on virtualization that enables rapid scaling of resources, the Iaas service includes storage, vehicle monitoring web application also based on virtualization and network virtualization.Fig.1 summarizes the Remote Vehicle monitoring cloud computing service proposed in this paper.

\section{B. Remote Service Event Processing}

Remote service event processing describes how data is collected, analyzed, stored on database; it is also integrates access to the stored data by examining OBD connector MAC address associated to car user car identification profile. The Remote service event processing consists of real time processing, integration and secure access and monitoring.

1) Secure access and monitoring: The interaction between the vehicles with the remote processing is tied up to raise complex privacy. Global identification considerations are necessary to ensure that the sensors devices and the user car have authorized profile. In the implementation, we consider to use Bluetooth MAC address to set unique identity of the OBD scanner as long as it support Bluetooth capabilities to communicate with android smartphone. By authorizing the user, database in the cloud returns an access profile validation message back to the application. The acquaintance of the user and the Global vehicle identification is established at the time the remote diagnostics aftermarket software and the OBD diagnostic scanner are given to the car owner.

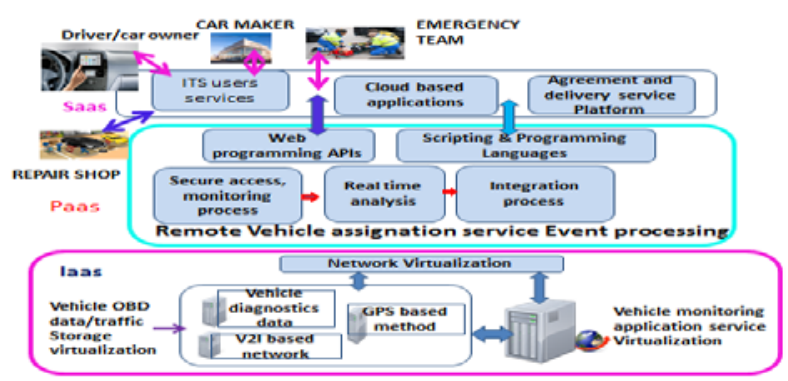

FIGURE I. REMOTE VEHICLE ASSIGNATION SERVICE CLOUD COMPUTING MODEL.

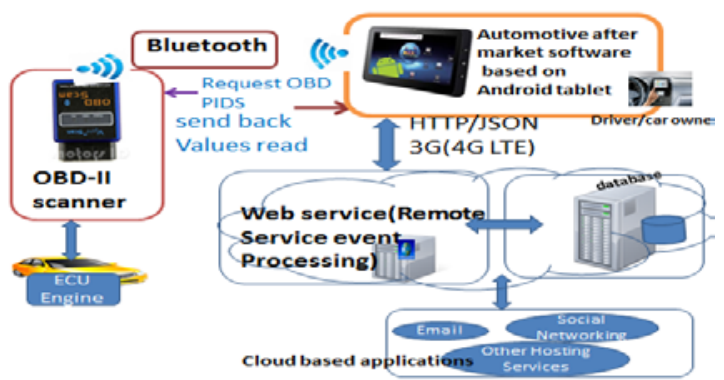

FIGURE II. REMOTE DATA ACQUISITION SYSTEM ON THE CLOUD.

2) Real time processing consists of collecting data from the vehicle on the OBD connector paired with the remote diagnostics application based on android platform. While data is uploaded via 3G or 4G LTE and HTTP protocol, data is transferred over internet in JSON exchange format for easily processing on the web service. The analysis takes place in web service by integrates a real time check based on SQL query to ensure data collected are sent from the proper car user. Advanced queries still performed and start triggers a predefined task to the dashboard based web application and to user car in case an abnormal or MIL (Malfunction indicator lamp) occurs for instance.

3) The integration event refers to the capacity to set up a list of actions to be taken in consideration and issued consecutive tasks in order to avoid or a fault to occur. Take example when the over speed is triggered, the emergency service team or the police near the located receive an alert that preventing that in an over speed is recorded. Those actions enable to integrate more and more actions to improve safety, reduced congestion, and improved energy efficient for instance. Fig. 2summarizes the full process that lead the implementation of the remote vehicle Diagnostics software as a service.

\section{CASE STUDY REMOTE VEHICLE DIAGNOSTICS SOFTWARE AS A SERVICE OFFERED AFTER CAR MARKET}

Remote vehicle Diagnostics Software as a service is used by the vehicle owner via his smartphone device, he can start use it by connecting the Bluetooth OBDII scan tool adapter into OBD-II car's connector.

\section{A. Data Acquisition and Storage}

Once the connection with the android based smartphones is established, it starts request data and the action is performed in background services. Fig.3 shows login before requesting engine performance and values uploaded into database. Vehicle 
OBD-II diagnosis data that have collected, along with the global vehicle identification number loaded from android smartphone's memory after the user has registered them within the application and vehicle location (latitude, longitude) that corresponds to the current position of the vehicle provided by the GPSare then saved in the databaselocates in automotive industry's datacenter in the format of JSON objects throughHTTP protocol. Fig. 4 shows a screen shot while the car owner requests from the cloud on-board diagnostics saved during his trip.

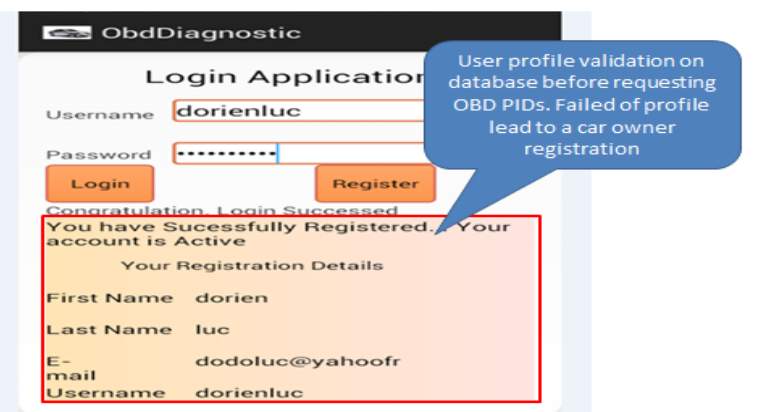

FIGURE III. VALIDATION CAR USER PROFILE ON CLOUD BEFORE REQUESTING OBD-PIDS.

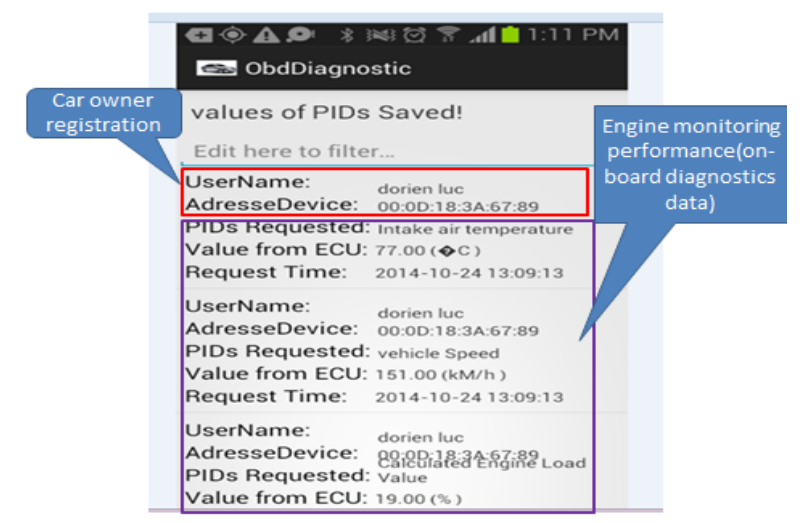

FIGURE IV. REQUEST OF ON-BOARD DIAGNOSTICS DATA SAVED ON THE CLOUD BY THE CAR OWNER.

\section{B. Car User Front End Web Portal for Monitoring}

The main idea of user-friendly front end web portal is to track down; monitor on board diagnostics [13], data that are already uploaded periodically from android based smartphone from different car owners in the cloud. The front web page allows also vehicle owners to have an idea of where they have been by analyzing Google maps which displays the path they took via various markers that correspond to the various locations provided by the global Positioning System (GPS). Fig. 5 describes the information available on the web front web portal accessible by the car user meanwhile he is not driving his car for instance at office or at home or any time he wants a car repair contacts him for more details about value uploaded on the remote database.

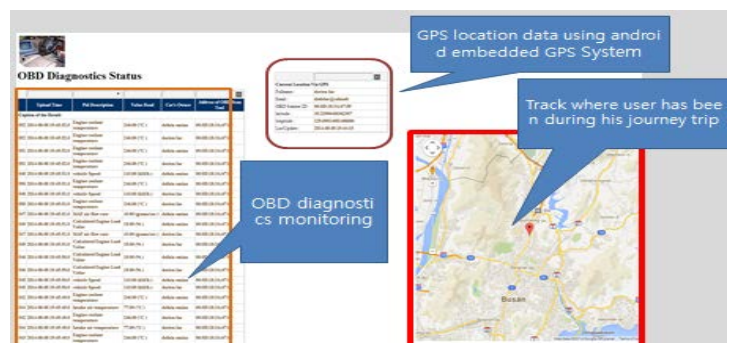

FIGURE V. CAR USER-FRIENDLY FRONT END WEB PORTAL.

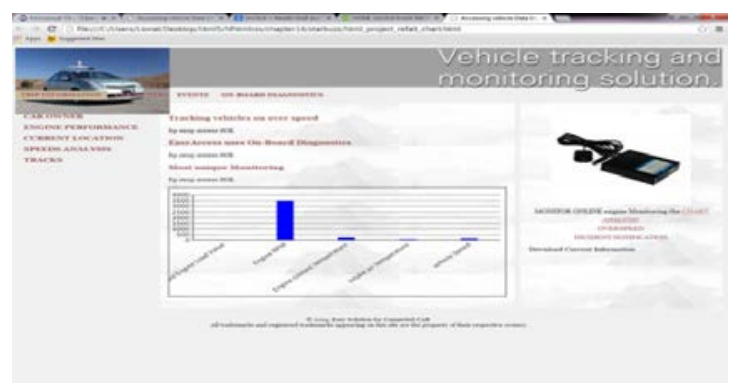

FIGURE VI.

\section{CAR MANUFACTURER-FRIENDLY FRONT END} WEB PORTAL.

\section{Car Manufacturer Web Portal}

Fig. 6 shows a screenshot of the car manufacturer dashboard that is used to display and monitoring on-board diagnostics for a particular mark of vehicle sold on the market. The dashboard has several options for displaying the following information :( 1)carowner information, (2)Engineperformance, (3) Current location, (4) Speed Analysis, (5) tracking OBDPIDs using charts.

\section{CONCLUSION}

In this paper, we argue that concerns about uprightness of data from traffic and on-board diagnostics are a major step for vehicle owners, authorities and businesses looking to take up cloud computing that enables value-added ,external applications and others services. We present a cloud computing which enables data remote vehicle assignation service event processing. A remote vehicle diagnostics software as a service attests the concept of vehicle to cloud capable of collecting diagnostics data. Our next purpose is to implement a fully prototype to evaluate others value-added services.

\section{ACKNOWLEDGMENT}

This research was supported by the Brain Busan 21 Project in 2014 and Nurimaru R\&BD Project (Busan IT Industry Promotion Agency) in 2014.

\section{REFERENCES}

[1] Leonard Kleinrock."Nomadic Computing -An opportunity”,CCR-4/95

[2] James O'Kane, Robert Trimble," Automotive cockpit modularity: migration issues for local tier 1 suppliers"

[3] JukkaAhola."Vehicle services opportunities benefit from the cloud”,Applying cloud technologies for business Magazine,page78-79

[4] Honda announces Android-based in-car infotainment system, http://www.androidos.in/2014/10/honda-announces-android-based-carinfotainment-system/

[5] SairaGillani,Imran Khan, Shahid Qureshi, Amir Qayyum, "Vehicular Ad Hoc Network(VANET):Enabling secure and Efficient Transportation System”. 
[6] Pyungsun Park, HongbinYim, Heeseok Moon, Jaeil Jung,” An OSGi Based In-Vehicle Gateway Platform Architecture for Improved Sensor Extensibility and Interoperability”, 2009 33rd Annual IEEE International Computer Software and Applications Conference, pp140-147.

[7] Jabar $\mathrm{H}$ Yousif, Cloud Computing and Accident Handling Systems,International Journal Applications(0975-8887),Vol.63N19,pp21-26,February 2013.

[8] What is cloud services?webopedia,http://www.webopedia.com/TERM/C/cloud_service s.html

[9] [Jin Wang1, 2, Jinsong Cho1, Sungyoung Lee1, TinghuaiMa,"Real Time Services for future Cloud Computing Enabled vehicle Networks".

[10] M. R. Martínez Torres, C. Díaz-Fernández, S.L. Toral, F. Barrero, F. Cort "Identification of added Value ITS Services using Concept Mapping Techniques",Proceedingd of the 12th International IEEE Conference on Intelligent Transportation Systems,St.Louis,MO,USA,October 3-7, 2009,page 54-59

[11] Peter H Jesty, Angela Spence, "Extend FRAMEwork architecture for cooperative systems", Contractual date of delivery, August 2011

[12] International telecommunication Union,ITU-T Focus Group on Driver Distraction, "Report on vehicle-to-Applications Communications Interface"

[13] Wikipedia,OBDIIPIDs, http://en.wikipedia.org/wiki/OBD-II_PIDs. 